\title{
Effect of a Mediterranean Diet during Pregnancy on Fetal Growth and Preterm Delivery: Results From a French Caribbean Mother-Child Cohort Study (TIMOUN)
}

\author{
Lauren Saunders, ${ }^{a}$ Laurence Guldner, ${ }^{\mathrm{b}}$ Nathalie Costet, ${ }^{\mathrm{a}}$ Philippe Kadhel, ${ }^{\mathrm{a}, \mathrm{c}}$ Florence Rouget ${ }_{1}^{\mathrm{a}}$ Christine Monfort, ${ }^{\mathrm{a}}$ \\ Jean-Pierre Thomé, ${ }^{d}$ Luc Multigner, ${ }^{a}$ Sylvaine Cordier ${ }^{\mathrm{a}}$ \\ ${ }^{a}$ Team of Epidemiological Research on Environment, Reproduction and Development, National Institute for Health and \\ Medical Research (INSERM U1085), Rennes \\ ${ }^{b}$ Department of Environmental Health, French Institute for Public Health Surveillance (InVS), St-Maurice \\ ${ }^{c}$ Gynecology and Obstetric Unit, CHU Pointe à Pitre/Abymes, Guadeloupe, French West Indies, France \\ ${ }^{d}$ Center for Analytical Research and Technology, Liege University, Liege, Wallonia, Belgium
}

\begin{abstract}
Background: Recent studies suggest that a Mediterranean dietary pattern during pregnancy may influence pregnancy outcomes. The aim of this study was to evaluate the effect of adherence to a Mediterranean diet (MD) during pregnancy on fetal growth restriction (FGR) and preterm delivery (PTD) in a French Caribbean island where the population is largely of African descent and presents dietary patterns similar to MD.

Methods: Using data from the TIMOUN Mother-Child Cohort Study conducted in Guadeloupe (French West Indies) between 2004 and 2007, we analysed data for 728 pregnant women who delivered liveborn singletons without any major congenital malformations. Degree of adherence to MD during pregnancy was evaluated with a semi-quantitative food frequency questionnaire based on nine dietary criteria. Multiple logistic regression models were used to analyse birth outcomes while taking potential confounders into account.

Results: Overall there was no association between MD adherence during pregnancy and the risk of PTD or FGR. However, pre-pregnancy body mass index was a strong effect modifier, and MD adherence was associated with a decreased risk of PTD specifically in overweight and obese women (adjusted odds ratio 0.7, 95\% confidence interval 0.6, 0.9) (P heterogeneity <0.01).

Conclusions: These results suggest that Caribbean diet during pregnancy may carry some benefits of MD and may contribute to reduce the risk of PTD in overweight and obese pregnant women.
\end{abstract}

Keywords: Mediterranean diet, preterm birth, fetal development, French West Indies.

Dietary patterns during pregnancy may have a considerable influence on fetal growth and the future health of children. Since the early 1990s, several studies have shown the beneficial role of some specific food groups (such as fish, fruits, vegetables and dairy products) and nutrients (such as omega-3 fatty acids, vitamins and minerals) on pregnancy and infant health-related outcomes. ${ }^{1-4}$ Some other food categories such as processed meat and sweet drinks ${ }^{5}$ were shown to have a detrimental role. Such studies usually did not consider the maternal diet as a whole and ignored the existing food and nutrient interactions that define the

\section{Correspondence:}

Sylvaine Cordier, INSERM, UMR1085, IRSET, Campus de Beaulieu, Bâtiment 13, 263 avenue du Général Leclerc, Rennes, 35042 France.

E-mail: sylvaine.cordier@inserm.fr global diet of the women. ${ }^{6}$ This limitation has been addressed using factor analysis to identify dietary patterns empirically derived from the observed consumptions within the population under study or based on a priori hypotheses (referring to dietary recommendations or adherence to presumably beneficial dietary patterns).

Recently, several mother-child cohorts have examined the potential benefits of adherence to a traditional Mediterranean dietary pattern (characterised by a high monounsaturated/saturated fat ratio, moderate consumption of milk and dairy products and of ethanol, high consumption of vegetables, fruits, legumes and grains, and low consumption of meat and meat products) ${ }^{7,8}$ during pregnancy on pregnancy outcomes and child development. Adherence to a Mediterranean diet (MD) during pregnancy was 
associated with reduced risk of preterm delivery (PTD) in Denmark ${ }^{9}$ but not in Norway ${ }_{1}^{10}$ and of spina bifida malformations. ${ }^{11}$ Low adherence to an MD in early pregnancy was associated with decreased intrauterine size with a lower placental and birthweight, ${ }^{12}$ while high adherence might modify the detrimental effect of smoking on birth size..$^{13}$ A reduction in the risk of asthma and atopy in childhood has been observed in association with high level of adherence to MD during pregnancy in Spain but not in a crosssectional study in Mexico. ${ }^{14}$

Until now, most investigations of the impact of the MD in pregnant women have studied Northern European (such as Danish and Norwegian) and Mediterranean populations (Spanish and Greek). Our study focuses on the population of the French West Indies, where most of the population is of African descent, and where rates of PTD and low birthweight are high compared with the French mainland. ${ }^{15}$ Dietary patterns observed in this population have several characteristics of an MD, including high intakes of fruits, vegetables, fish and crustaceans, and low intakes of cheese and sweets.

The objective of this study was to evaluate the impact of MD adherence during pregnancy on fetal growth restriction (FGR) and PTD in a large population-based mother-child cohort study (TIMOUN) set up in Guadeloupe in 2004.

\section{Methods}

\section{Population and study design}

The study population consisted of pregnant women enrolled in the Guadeloupean prospective motherchild cohort study TIMOUN, from November 2004 to December 2007. Women who had been living in Guadeloupe for at least 3 years were invited to participate in the study during the third-trimester prenatal visit at a Guadeloupe public hospital (university and local hospital) or a local antenatal care clinic. Doctors presented the objectives of the study, and each participant provided written informed consent. At inclusion, women responded to a detailed questionnaire about their sociodemographic characteristics, medical and obstetric history, occupation and life style during in-person interviews, and specially trained midwives collected medical data. Data collected at delivery included the newborn's health status and details of delivery. A food frequency questionnaire (FFQ), asking about usual dietary intake during this pregnancy, was also administered shortly after delivery.

From 2004 to 2007, the study included 1068 women, $820(77 \%)$ of whom completed the FFQ. Twenty-three women were further excluded from the analysis because the FFQ or outcome information was incomplete, and 25 were excluded for implausible values for total energy intake (outside the range of 3347$25104 \mathrm{~kJ} / \mathrm{d}$ ). Finally, the analysis was restricted to women with a liveborn singleton pregnancy without major congenital malformations. The final analysis was based on 728 pregnant women. All procedures were approved by the Guadeloupean Advisory Committee on the Protection of Persons in Biomedical Research.

\section{Dietary assessment}

Women were interviewed about their diet during the current pregnancy with a validated semi-quantitative FFQ including 214 food items administered by trained interviewers in the days following delivery. For each food item, women declared their usual frequency and portion size of intake from which we estimated a daily intake (g/day) by multiplying the intake frequency by the portion size (details of the construction of the questionnaire are given in Supporting Information File S1).

The validity and reproducibility of the FFQ were evaluated by several methods (Supporting Information File S1). The FFQ-based method showed a good reproducibility and validity when compared with a diary-based method in a validation sample $(73.0 \%$ of total energy intakes classified in the same or adjacent quintile by both methods). Correlations between fatty acid intake estimates from FFQ and maternal blood concentrations of fatty acids were on the order of those previously reported in the literature.

To evaluate the degree of adherence to the traditional MD during pregnancy, we used a scale constructed by Trichopoulou et al. ${ }^{16}$ revised to include fish intake. ${ }^{17}$ Each of the 214 items was classified among the nine categories of the Mediterranean scale (vegetables, legumes, fruits and nuts, cereals, fish, meat and poultry, dairy products, alcohol and fat). As done previously in studies among pregnant women, ${ }^{13}$ the classification of food groups as beneficial or detrimental differed from the traditional MD in the general population: we presumed dairy products to be 'protective' components ${ }^{4}$ and alcohol consumption to be 
'detrimental'. ${ }^{18}$ For beneficial components (vegetables, legumes, fruits and nuts, cereals, fish and dairy products), women with daily intake (g/day) below the (cohort-specific) median were assigned a value of 0 , and women with consumption at or above the median were assigned a value of 1 . For components presumably detrimental (meat, poultry and alcohol), women with daily intake below the median were assigned a value of 1 , and women with intake at or above the median were assigned a value of 0 . The fat components were expressed as the ratio of monounsaturated lipids [monounsaturated fatty acids (MUFA)] to saturated lipids [saturated fatty acids (SFA)]. The MD score (MDS) was calculated by the sum of the values assigned to the nine components of the MDS and ranged from 0 (minimal adherence to the traditional MD) to 9 (maximal adherence).

\section{Assessment of fetal growth and PTD}

Birthweight was extracted from the pediatricians' records at birth. Gestational age was established by obstetricians on the basis of the last menstrual period and corrected by ultrasound measurement recorded in the first trimester of pregnancy. PTD was defined as any birth occurring before 37 weeks of gestation. FGR was defined according to the statistical method described by Ego et al. ${ }^{19}$ and was determined by comparing actual birthweight with the expected birthweight, predicted by a multiple linear regression including known determinants of growth such as age, weight and height of the mother, parity, gestational age and sex. This definition therefore takes into account the constitutional characteristics of the mother of the child. Newborns with a weight below the tenth percentile of the predicted distribution were considered growth-restricted.

\section{Statistical analysis}

The outcome variables of interest were PTD and FGR as categorical variables. The primary exposure of interest was MD adherence during pregnancy measured by the MDS. The score was categorised into three levels of adherence, as previously done by Trichopoulou et al..$^{20}$ and Chatzi et al. ${ }^{13}$ : low adherence (MDS $\leq 3)$, medium adherence (MDS $=4$ or 5$)$, high adherence (MDS $\geq 6$ ). Multiple logistic regressions were used to analyse dichotomous birth outcomes. Regression models included covariates that have an established or potential association with outcome variables and MD adherence: maternal region of birth (Guadeloupe/Martinique, other Caribbean islands, Europe), marital status (married or in couple, single or single living with own family), pre-pregnancy body mass index (BMI; in $\mathrm{kg} / \mathrm{m}^{2}$ ) (underweight or normal: $<25$, overweight or obese: $\geq 25$ ), maternal education (low: $<5$, medium: 5-12, high: $>12$ years of education), enrolment site (university hospital, local antenatal care clinic, local hospital), average weekly weight gain during pregnancy (in $\mathrm{g} /$ week) $(<340, \geq 340)$ and smoking during pregnancy (yes, no). Gestational weight gain (GWG) was computed as average weekly weight gain to take into account varying gestational week at measurement. Adherence to IOM recommendations ${ }^{21}$ was assessed in three categories (low, normal or high weight gain during pregnancy) according to pre-pregnancy BMI. All analyses were further adjusted for total energy intake during pregnancy, categorised into tertiles (in kJ/day) (<9243, 923412 410, >12 410). Effect modifications according to prepregnancy BMI and infant sex were tested and considered significant when Wald test $P$ value $<0.20$. Medically indicated and spontaneous PTD were also studied separately. Analyses were performed with Statistical Analysis Systems statistical software package version 9.3 (SAS Institute, Cary, NC, USA).

\section{Results}

The identified diet was characterised by high intakes of fruits and nuts (median $501 \mathrm{~g} / \mathrm{d}$ ), dairy products (median $331 \mathrm{~g} / \mathrm{d}$ ), vegetables (median $283 \mathrm{~g} / \mathrm{d}$ ), cereals (median $272 \mathrm{~g} / \mathrm{d}$ ) and fish (median $88 \mathrm{~g} / \mathrm{d}$ ) (Table 1). The mean MDS was 4.9 ( \pm 1.6$)$ (median: 5, interquartile range: 4-6). Twenty per cent of the women had low adherence to MD (MDS $\leq 3), 42 \%$ medium adherence $(4 \leq \mathrm{MDS} \leq 5)$ and $37 \%$ high adherence (MDS $\geq 6$ ). Women with high MDS differed from those with low MDS by higher intakes of fish $(\times 4)$, vegetables $(\times 3)$ and legumes $(\times 3)$, fruits and nuts $(\times 2)$. Overweight and obese women did not differ from underweight/normal regarding any food group intake.

Women included in the analysis had a mean age ( \pm standard deviation) of $31( \pm 7)$ years (Table 2$)$. Most of them were born in Guadeloupe or Martinique (78.4\%) and were enrolled at the University Hospital (73.8\%). Of the 728 women, only 399 (56.2\%) were living in couple. Sixty-eight per cent had a medium 


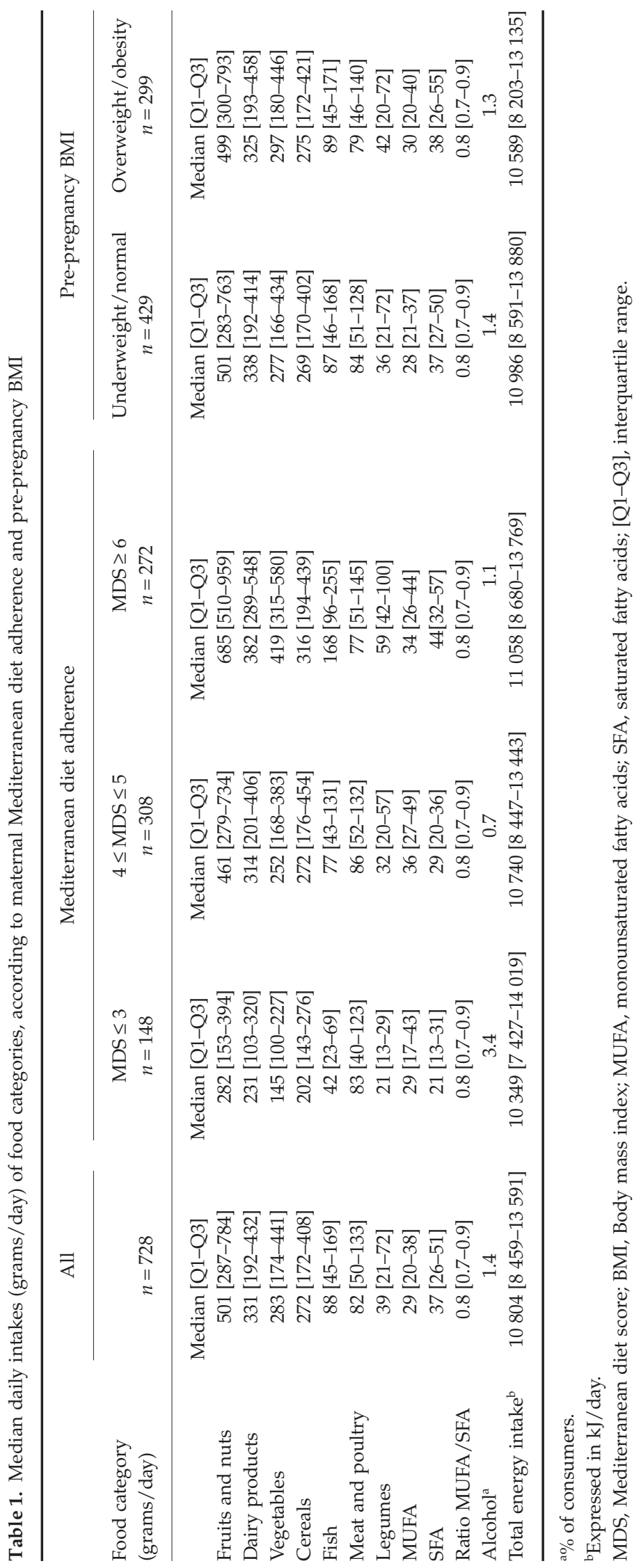


Table 2. Mediterranean Diet Score according to maternal sociodemographic and lifestyle characteristics, preterm delivery and fetal growth restriction $(n=728)$

\begin{tabular}{|c|c|c|c|c|c|}
\hline & \multirow[b]{2}{*}{$n$} & \multirow[b]{2}{*}{$\%$} & \multicolumn{2}{|c|}{ MDS } & \multirow[b]{2}{*}{$P$} \\
\hline & & & Mean & SD & \\
\hline Maternal place of birth & & & & & 0.03 \\
\hline Guadeloupe/Martinique & 571 & 78.4 & 5.0 & 1.6 & \\
\hline Other Caribbean islands & 72 & 9.9 & 4.5 & 1.5 & \\
\hline Europe & 85 & 11.7 & 4.9 & 1.6 & \\
\hline Marital status & & & & & 0.64 \\
\hline Single & 176 & 24.8 & 5.0 & 1.5 & \\
\hline Married or in couple & 399 & 56.2 & 5.0 & 1.6 & \\
\hline Living with own family & 135 & 19.0 & 4.8 & 1.6 & \\
\hline Maternal Education (years) & & & & & 0.30 \\
\hline Low $(<5)$ & 36 & 5.0 & 4.6 & 1.8 & \\
\hline Medium (5-12) & 492 & 67.6 & 4.9 & 1.5 & \\
\hline High $(>12)$ & 200 & 27.5 & 5.0 & 1.6 & \\
\hline Enrolment site & & & & & $<0.01$ \\
\hline University hospital & 537 & 73.8 & 5.1 & 1.5 & \\
\hline Local antenatal care clinic & 126 & 17.3 & 4.7 & 1.7 & \\
\hline Local hospital & 65 & 8.9 & 4.5 & 1.6 & \\
\hline Pre-pregnancy BMI $\left(\mathrm{kg} / \mathrm{m}^{2}\right)$ & & & & & 0.56 \\
\hline Underweight & 50 & 6.9 & 5.3 & 1.8 & \\
\hline Normal & 379 & 52.0 & 4.9 & 1.6 & \\
\hline Overweight & 163 & 22.4 & 5.1 & 1.5 & \\
\hline Obese & 136 & 18.7 & 4.9 & 1.5 & \\
\hline $\begin{array}{l}\text { Weight gain during pregnancy } \\
\qquad(\mathrm{g} / \text { week })\end{array}$ & & & & & 0.18 \\
\hline$<340$ & 372 & 51.1 & 5.0 & 1.5 & \\
\hline$\geq 340$ & 356 & 48.9 & 4.9 & 1.6 & \\
\hline $\begin{array}{l}\text { Energy intake during } \\
\text { pregnancy }(\mathrm{kJ} / \mathrm{d})\end{array}$ & & & & & 0.73 \\
\hline$<9234$ & 241 & 33.1 & 4.9 & 1.7 & \\
\hline $9234-12410$ & 239 & 32.8 & 5.0 & 1.5 & \\
\hline$>12410$ & 248 & 34.1 & 5.0 & 1.5 & \\
\hline Smoking during pregnancy & & & & & 0.60 \\
\hline No & 689 & 94.6 & 4.9 & 1.6 & \\
\hline Yes & 39 & 5.4 & 5.1 & 1.3 & \\
\hline Alcohol during pregnancy & & & & & 0.13 \\
\hline No & 706 & 97.0 & 5.0 & 1.5 & \\
\hline Yes & 22 & 3.0 & 4.4 & 1.6 & \\
\hline Sex of the newborn & & & & & 0.78 \\
\hline Male & 370 & 50.8 & 5.0 & 1.6 & \\
\hline Female & 358 & 49.2 & 4.9 & 1.6 & \\
\hline Preterm delivery & & & & & 0.27 \\
\hline No & 621 & 85.3 & 5.0 & 1.6 & \\
\hline Yes & 107 & 14.7 & 4.8 & 1.5 & \\
\hline Fetal growth restriction & & & & & 0.85 \\
\hline No & 635 & 87.2 & 4.9 & 1.6 & \\
\hline Yes & 93 & 12.8 & 5.0 & 1.5 & \\
\hline
\end{tabular}

MDS, Mediterranean diet score; SD, standard deviation; BMI, body mass index. education level and $27.5 \%$ had a high education level. Fifty-two per cent had a normal pre-pregnancy BMI, $6.9 \%$ were underweight, $22.4 \%$ were overweight and $18.7 \%$ were obese. Women born in other Caribbean Islands had significantly lower adherence to MD, and those enrolled at the University hospital had significantly higher MDS.

The mean birthweight was 3095 ( \pm 508$)$ g. Preterm deliveries accounted for $14.7 \%$ of all births (interquartile range: $37-40$ weeks), and $52.8 \%$ of them were spontaneous. FGR was diagnosed in 93 newborns $(12.8 \%)$. Preterm $(14 \%, 17 \%, 13 \%)$ and FGR $(11 \%, 12 \%, 14 \%)$ prevalence was similar amongst the three categories of MDS.

Table 3 reports the associations between MD adherence during pregnancy and PTD, stratified for prepregnancy BMI and infant sex. A non-significant decrease in the risk of PTD in association with MDS was observed in the entire sample of women (adjusted odds ratio (aOR) $0.9,95 \%$ confidence interval (CI) 0.8 , 1.0). Adherence to the MD during pregnancy was associated with a statistically significant decrease in the risk of PTD in overweight and obese women (aOR 0.7, $95 \%$ CI $0.6,0.9) \quad(\mathrm{P}$ heterogeneity $<0.01$ ) especially among those with low or normal weight gain during pregnancy (Supporting Information File S2). There was no correlation between MDS score and GWG in overweight and obese women. We also observed an almost significant reduced risk of PTD among baby girls (aOR $0.8,95 \%$ CI 0.7, 1.0), but the effect heterogeneity was not significant (P heterogeneity $=0.21$ ).

$\mathrm{MD}$ adherence was not significantly associated with the risk of delivering an infant with FGR, but we observed a significant interaction term (P heterogeneity $=0.03$ ) and a non-significantly decreased risk of FGR associated with MDS among underweight and normal-weight women only (aOR $0.8,95 \%$ CI $0.6,1.0$ ) (Table 4). The same pattern was observed when analysis was restricted to FGR among term births.

Sensitivity analyses confirm these findings, showing a statistically significant decrease in the risk of PTD in relation with MD adherence among overweight and obese women when taking into account infants without FGR exclusively, and identical estimates among indicated preterm deliveries. However, the association between MD adherence and the risk of PTD was not found significant among spontaneous preterm deliveries (probably due to a lower proportion of overweight and obese women in this group) (Supporting Information File S2). 
Table 3. Odds ratios of preterm delivery per unit of Mediterranean Diet Score, stratified for pre-pregnancy BMI and infant sex $(n=728)$

\begin{tabular}{|c|c|c|c|c|c|c|c|}
\hline & $\mathrm{N}$ & $\begin{array}{l}\text { PTD } \\
\text { cases }\end{array}$ & $\begin{array}{c}\text { Unadjusted } \\
\text { OR }\end{array}$ & $95 \% \mathrm{CI}$ & $\begin{array}{c}\text { Adjusted } \\
\text { OR }\end{array}$ & $95 \% \mathrm{CI}$ & P-value $e^{a}$ \\
\hline Non stratified model $^{\mathrm{b}}$ & 728 & 107 & 0.9 & $0.8,1.1$ & 0.9 & $0.8,1.0$ & \\
\hline Model stratified for $\mathrm{BMI}^{\mathrm{c}}$ & & & & & & & $<0.01$ \\
\hline Underweight/normal & 429 & 56 & 1.1 & $0.9,1.3$ & 1.1 & $0.9,1.3$ & \\
\hline Overweight/obesity & 299 & 51 & 0.7 & $0.6,0.9$ & 0.7 & $0.6,0.9$ & \\
\hline Model stratified for infant sex ${ }^{b}$ & & & & & & & 0.20 \\
\hline Male & 370 & 58 & 1.0 & $0.8,1.2$ & 1.0 & $0.8,1.2$ & \\
\hline Female & 358 & 49 & 0.9 & $0.7,1.0$ & 0.8 & $0.7,1.0$ & \\
\hline
\end{tabular}

aP values for the effect heterogeneity test.

${ }^{b}$ Adjusted for maternal place of birth, marital status, pre-pregnancy BMI, maternal education, enrolment site, weight gain during pregnancy, energy intake and maternal smoking during pregnancy $(N=710$ with complete data).

'Adjusted for maternal place of birth, marital status, maternal education, enrolment site, weight gain during pregnancy, energy intake and maternal smoking during pregnancy ( $N=710$ with complete data).

PTD, preterm delivery; OR, odds ratio; CI, confidence interval; BMI, pre-pregnancy body mass index.

\section{Comments}

This study shows a significant reduction in the risk of PTD among pregnant overweight and obese women who ate a Mediterranean-type diet during their pregnancy.
Its potential beneficial effects on health, especially its potential to reduce the risks of cancer and cardiovascular and chronic diseases, have made the MD a topic of major interest today. ${ }^{22-24}$ Despite the common characteristics of this dietary pattern (high consumption of fruit, vegetables, legumes and grains, moderate

Table 4. Odds ratios of fetal growth restriction per unit of Mediterranean Diet Score, stratified for pre-pregnancy BMI and infant sex

\begin{tabular}{|c|c|c|c|c|c|c|c|}
\hline & $N$ & $\begin{array}{l}\text { FGR } \\
\text { cases }\end{array}$ & $\begin{array}{c}\text { Unadjusted } \\
\text { OR }\end{array}$ & $95 \% \mathrm{CI}$ & $\begin{array}{l}\text { Adjusted } \\
\text { OR }\end{array}$ & $95 \% \mathrm{CI}$ & P-value ${ }^{a}$ \\
\hline \multicolumn{8}{|l|}{ All births } \\
\hline Non stratified model ${ }^{\mathrm{b}}$ & 728 & 93 & 1.0 & $0.9,1.2$ & 1.0 & $0.8,1.1$ & \\
\hline Model stratified for $\mathrm{BMI}^{\mathrm{c}}$ & & & & & & & 0.03 \\
\hline Underweight/normal & 429 & 42 & 0.9 & $0.7,1.1$ & 0.8 & $0.7,1.0$ & \\
\hline Overweight/obesity & 299 & 51 & 1.1 & $0.9,1.4$ & 1.2 & $0.9,1.5$ & \\
\hline Model stratified for infant sex ${ }^{b}$ & & & & & & & 0.69 \\
\hline Male & 370 & 39 & 1.0 & $0.8,1.2$ & 0.9 & $0.7,1.1$ & \\
\hline Female & 358 & 54 & 1.0 & $0.9,1.2$ & 1.0 & $0.8,1.2$ & \\
\hline \multicolumn{8}{|l|}{ Among term births } \\
\hline Non-stratified model ${ }^{\mathrm{b}}$ & 621 & 68 & 1.0 & $0.9,1.2$ & 1.0 & $0.8,1.2$ & \\
\hline Model stratified for $\mathrm{BMI}^{\mathrm{c}}$ & & & & & & & 0.05 \\
\hline Underweight/normal & 373 & 31 & 0.9 & $0.7,1.2$ & 0.8 & $0.7,1.1$ & \\
\hline Overweight/obesity & 248 & 37 & 1.2 & $0.9,1.5$ & 1.3 & $0.9,1.7$ & \\
\hline Model stratified for infant sex ${ }^{b}$ & & & & & & & 0.92 \\
\hline Male & 312 & 26 & 1.1 & $0.8,1.4$ & 0.9 & $0.7,1.3$ & \\
\hline Female & 309 & 42 & 1.0 & $0.8,1.3$ & 1.0 & $0.8,1.2$ & \\
\hline
\end{tabular}

${ }^{a} P$ values for the effect heterogeneity test.

${ }^{b}$ Adjusted for maternal place of birth, marital status, pre-pregnancy BMI, maternal education, enrolment site, weight gain during pregnancy, energy intake and maternal smoking during pregnancy $(N=710$ with complete data in all births, $N=609$ in term births only).

cAdjusted for maternal place of birth, marital status, education level, enrolment site, weight gain during pregnancy, energy intake and maternal smoking during pregnancy ( $N=710$ with complete data in all births, $N=609$ in term births only).

FGR, fetal growth restriction; OR, odds ratio; CI, confidence interval; BMI, body mass index. 
consumption of fish, dairy products and alcohol, low meat intake), it is subject to some regional variations associated with sociocultural, population and geographic differences, ${ }^{25,26}$ as well as with changes and different influences associated with the globalisation process. ${ }^{27}$ These factors make it difficult to establish a specific definition. To these difficulties we must add the nutritional specificities of pregnant women, who should avoid alcohol consumption during pregnancy because of its harmful impact on fetal development and should consume more dairy products than usual because of their increased calcium requirements. Chatzi et al. ${ }^{13}$ showed heterogeneity between Spain and Greece, countries traditionally known for an MD: intake of fish and dairy products was higher in the Atlantic region of Spain and fruit and nut intake higher in Crete. They reported a lower risk of delivering FGR infants for women with high MD adherence in the Mediterranean-INMA study (Spain), but not in the Atlantic-INMA or the RHEA cohort (Greece). If we compare these observations with the dietary pattern of our Guadeloupian population, we observe higher daily consumption of vegetables $(283 \mathrm{~g} /$ day), fruit and nuts (501 g/day) and fish ( $88 \mathrm{~g} /$ day) in this cohort than among the women questioned in the Atlantic Spain, Mediterranean Spain or Greek cohorts. Although this dietary profile does not truly match any of these three particular patterns, the high fruit and nut intake and moderate dairy product consumption are similar to that of the Greek cohort, while the high fish consumption is closer to the pattern of the Atlantic region of Spain.

Very few studies have examined the effect on length of gestation and fetal growth of eating an MD during pregnancy. ${ }^{9,10,12,13}$ To our knowledge, only a few recent prospective studies have shown an association between this type of diet and the risk of PTD. In 2005, a randomised study in a cohort of Norwegian women showed a $90 \%$ reduction in the PTD rate among women who ate a low-cholesterol diet and reduced their SFA intake during pregnancy. ${ }^{28}$ In that study, the women in the intervention group were supposed to follow a diet including greater intake of fish, vegetable oils (olive oil in particular), fruit and vegetables, nuts, and a reduction in meat, butter, margarine, cream and other fat-rich dairy products, together with a reduction in coffee. Among the 141 women included in the intervention group, 1 woman $(0.7 \%)$ gave birth to a preterm child, compared with $11(7.4 \%)$ of the 149 women in the control group.
In an observational cohort of 35530 Danish women published in 2008, Mikkelsen et al. ${ }^{9}$ showed a $72 \%$ reduction in the risk of early preterm delivery (defined as birth before 35 weeks of gestation) associated with following an MD during pregnancy. The women meeting all the following criteria were considered to be following an MD correctly: consumption of fish at least twice a week, consumption of olive oil, high consumption of fruit and vegetables (at least five a day), meat eaten a maximum of twice a week and a maximum of two cups of coffee a day.

Similarly, an MD throughout pregnancy appeared to reduce the risk of PTD in our study. According to Khoury et al., ${ }^{28}$ several hypotheses may explain this effect. Some factors included in this diet could contribute to this result, in particular, the n-3 (omega-3) polyunsaturated fatty acids (PUFA). Increased intake of PUFA together with a reduction in SFA could influence the levels of inflammatory cytokines, which are involved in the pathophysiological mechanisms of PTD. ${ }^{29}$ Despite some contradictory findings, several studies have shown an association between PUFA and the duration of gestation. ${ }^{30}$ Khoury et al. ${ }^{28}$ also suggest that some micronutrients, such as vitamins $\mathrm{C}$ and $\mathrm{E}$, influence this increased duration of gestation; they also suggest that some dietary factors may play a role in reducing intrauterine bleeding, which is a known risk factor for PTD. Finally, according to Ness et al.,$^{31}$ there is probably an interaction between maternal blood levels of two folate isoforms and the risk of preterm delivery.

A novel finding in this study is that the beneficial effects of the MD on the risk of PTD were significantly greater among overweight and obese women compared with the normal and underweight women, although MDS score did not differ between these two categories. A pathophysiological perspective may explain this result. Excess weight is known to increase the risk of glucose intolerance and pregnancy-related diabetes. ${ }^{32}$ Moreover, it is recognised that glucose intolerance is associated with a shorter duration of gestation, independently of other known risk factors for PTD. ${ }^{33,34}$ An appropriate diet, including fewer simple carbohydrates, should result in better blood glucose regulation, which in turn should be associated with an increase in the duration of gestation, especially for obese women, who might be the first to benefit from this change. In our study, there is no indication that a mode of action of MD adherence through a reduction in GWG in overweight/obese women is 
acting, since no correlation was observed between MDS score and GWG.

Moreover, we observed a slight decreased risk of FGR associated with MDS among underweight and normal-weight women with a significant interaction term. This result is in accordance with a previous analysis within a large mother-child cohort in Spain that have showed a significantly lower risk of delivering an FGR infant for weight among women with high MD adherence. ${ }^{13}$

This study has numerous strengths. To our knowledge, it is the first prospective cohort study to explore the association between an MD in pregnant women and fetal growth and PTD in a Caribbean population of African descent. The few studies in this domain have been conducted principally in European Nordic or Mediterranean populations. The particularly high rates of PTD and low birthweight in the Caribbean population of African descent makes research into factors that might reduce these risks a public health priority. Data on food consumption come from an FFQ specific to the French West Indies (in particular, in terms of types of foods and methods of preparation and cooking) and validated by various methods described above. To minimise the response biases inherent in this type of questionnaire, a trained investigator administered it during a face-to-face interview. In our study, introducing an interviewer in the dietary assessment was relevant since the women's education level was low or medium for $72.6 \%$ of them, and some of them probably did not speak French fluently. FGR was defined according to an internal reference in view of the absence of reference curves adapted to this population. Midwives and paediatricians participating in this cohort study collected the fetal measurements at birth.

This study is nonetheless subject to some limitations. The PTD rate observed in our cohort of pregnant women in Guadeloupe is high (14.7\%), exceeding the estimates for the overseas districts and territories obtained from the French Perinatal Survey conducted in $2010(12 \%){ }^{15}$ The high recruitment at the Guadeloupe University Hospital (73.8\%) probably explains this difference since it favours inclusion of pregnant women with diseases during pregnancy, indications of induced delivery.

On the other hand, the FFQ was administered at the end of pregnancy and concerned the women's average diet throughout pregnancy. We therefore could not take into account the possible variations in diet during pregnancy or define a window of exposure during which the dietary pattern might be more important. A longitudinal study intended to identify dietary patterns among women throughout their pregnancy nonetheless shows that these variations are not significant. ${ }^{35}$ Finally, the intake estimates were completed retrospectively, after delivery. Accordingly, they could possibly have been influenced by the pregnancy outcome. This is nonetheless improbable because women with major pregnancy-related diseases were not included in the study; $99.1 \%$ of the PTD were moderately preterm, and the detailed questionnaire did not refer to any 'at risk' food, except for alcoholic beverages.

In conclusion, among women who were overweight or obese before pregnancy, the risk of PTD was significantly lower for those who followed an MD during pregnancy. This result, if confirmed, suggests a benefit of this type of diet during pregnancy and is especially interesting in the French West Indian population, which has a particularly elevated prevalence of obesity and PTD. Indeed, the multi-causal and complex nature of PTD makes its control particularly difficult. As a potentially modifiable contributor to PTD risk, setting up preventive dietary measures as part of the management of these overweight and obese pregnancies may have a substantial impact on the child's health status at birth and subsequent outcome.

\section{Acknowledgements}

This study was made possible by the support of the French National Health Directorate, French Ministry of Environment, French Agency for Environmental and Occupational Safety, National Agency for Research, French Institute for Public Health Surveillance, Regional Council of Guadeloupe. All authors saw and approved the final manuscript. The authors declare no conflict of interests.

\section{References}

1 Olsen SF. Consumption of marine n-3 fatty acids during pregnancy as a possible determinant of birth weight. A review of the current epidemiologic evidence. Epidemiologic Reviews 1993; 15:399-413.

2 Scholl TO, Hediger ML, Schall JI, Khoo CS, Fischer RL. Dietary and serum folate: their influence on the outcome of pregnancy. The American Journal of Clinical Nutrition 1996; 63:520-525. 
3 Brantsaeter AL, Haugen M, Samuelsen SO, Torjusen H, Trogstad L, Alexander J, et al. A dietary pattern characterized by high intake of vegetables, fruits, and vegetable oils is associated with reduced risk of preeclampsia in nulliparous pregnant Norwegian women. The Journal of Nutrition 2009; 139:1162-1168.

4 Brantsaeter AL, Olafsdottir AS, Forsum E, Olsen SF, Thorsdottir I. Does milk and dairy consumption during pregnancy influence fetal growth and infant birthweight? A systematic literature review. Food and Nutrition Research 2012; 56:20050.

5 Knudsen VK, Orozova-Bekkevold IM, Mikkelsen TB, Wolff $\mathrm{S}$, Olsen SF. Major dietary patterns in pregnancy and fetal growth. European Journal of Clinical Nutrition 2008; 62:463-470.

6 Sanchez-Villegas A, Brito N, Doreste-Alonso J, Nissensohn M, Henriquez P, Hermoso M, et al. Methodological aspects of the study of dietary patterns during pregnancy and maternal and infant health outcomes. A systematic review. Maternal and Child Nutrition 2010; 6 (Suppl. 2):100-111.

7 Trichopoulou A, Lagiou P. Healthy traditional Mediterranean diet: an expression of culture, history, and lifestyle. Nutrition Reviews 1997; 55:383-389.

8 Willett WC, Sacks F, Trichopoulou A, Drescher G, Ferro-Luzzi A, Helsing E, et al. Mediterranean diet pyramid: a cultural model for healthy eating. American Journal of Clinical Nutrition 1995; 61:1402S-1406S.

9 Mikkelsen TB, Osterdal ML, Knudsen VK, Haugen M, Meltzer HM, Bakketeig L, et al. Association between a Mediterranean-type diet and risk of preterm birth among Danish women: a prospective cohort study. Acta Obstetricia et Gynecologica Scandinavica 2008; 87:325-330.

10 Haugen M, Meltzer HM, Brantsaeter AL, Mikkelsen T, Osterdal ML, Alexander J, et al. Mediterranean-type diet and risk of preterm birth among women in the Norwegian Mother and Child Cohort Study (MoBa): a prospective cohort study. Acta Obstetricia et Gynecologica Scandinavica 2008; 87:319-324.

11 Vujkovic M, Steegers EA, Looman CW, Ocke MC, van der Spek PJ, Steegers-Theunissen RP. The maternal Mediterranean dietary pattern is associated with a reduced risk of spina bifida in the offspring. BJOG: An International Journal of Obstetrics and Gynaecology 2009; 116:408-415.

12 Timmermans S, Steegers-Theunissen RP, Vujkovic M, den Breeijen H, Russcher H, Lindemans J, et al. The Mediterranean diet and fetal size parameters: the Generation R Study. The British Journal of Nutrition 2012; 108:1399-1409.

13 Chatzi L, Mendez M, Garcia R, Roumeliotaki T, Ibarluzea J, Tardon A, et al. Mediterranean diet adherence during pregnancy and fetal growth: INMA (Spain) and RHEA (Greece) mother-child cohort studies. The British Journal of Nutrition 2012; 107:135-145.

14 Chatzi L, Kogevinas M. Prenatal and childhood Mediterranean diet and the development of asthma and allergies in children. Public Health Nutrition 2009; 12:1629-1634.
15 Blondel B, Kermarrec M (eds). Enquête nationale périnatale 2010 - Les naissances en 2010 et leur évolution depuis 2003. Paris: INSERM, 2011.

16 Trichopoulou A, Kouris-Blazos A, Wahlqvist ML, Gnardellis C, Lagiou P, Polychronopoulos E, et al. Diet and overall survival in elderly people. British Medical Journal 1995; 311:1457-1460.

$17 \mathrm{Hu}$ FB, Bronner L, Willett WC, Stampfer MJ, Rexrode KM, Albert CM, et al. Fish and omega-3 fatty acid intake and risk of coronary heart disease in women. Journal of the American Medical Association 2002; 287:1815-1821.

18 Patra J, Bakker R, Irving H, Jaddoe VW, Malini S, Rehm J. Dose-response relationship between alcohol consumption before and during pregnancy and the risks of low birthweight, preterm birth and small for gestational age (SGA)-a systematic review and meta-analyses. BJOG: An International Journal of Obstetrics and Gynaecology 2011; 118:1411-1421.

19 Ego A, Subtil D, Grange G, Thiebaugeorges O, Senat MV, Vayssiere C, et al. Customized versus population-based birth weight standards for identifying growth restricted infants: a French multicenter study. American Journal of Obstetrics and Gynecology 2006; 194:1042-1049.

20 Trichopoulou A, Costacou T, Bamia C, Trichopoulos D. Adherence to a Mediterranean diet and survival in a Greek population. New England Journal of Medicine 2003; 348:2599-2608

21 Siega-Riz AM, Gray GL. Gestational weight gain recommendations in the context of the obesity epidemic. Nutrition Reviews 2013; 71:S26-S30.

22 Panagiotakos DB, Pitsavos C, Polychronopoulos E, Chrysohoou C, Zampelas A, Trichopoulou A. Can a Mediterranean diet moderate the development and clinical progression of coronary heart disease? A systematic review. Medical Science Monitor 2004; 10:RA193-RA198.

23 de Lorgeril M, Salen P, Martin JL, Monjaud I, Boucher P, Mamelle N. Mediterranean dietary pattern in a randomized trial: prolonged survival and possible reduced cancer rate. Archives of Internal Medicine 1998; 158:1181-1187.

24 Sofi F, Cesari F, Abbate R, Gensini GF, Casini A. Adherence to Mediterranean diet and health status: meta-analysis. British Medical Journal 2008; 337:a1344.

25 Martinez-Gonzalez MA, Holgado B, Gibney M, Kearney J, Martinez JA. Definitions of healthy eating in Spain as compared to other European Member States. European Journal of Epidemiology 2000; 16:557-564.

26 Noah A, Truswell AS. There are many Mediterranean diets. Asia Pacific Journal of Clinical Nutrition 2001; 10:2-9.

27 Serra-Majem L, Trichopoulou A, Ngo de la Cruz J, Cervera P, Garcia Alvarez A, La Vecchia C, et al. Does the definition of the Mediterranean diet need to be updated? Public Health Nutrition 2004; 7:927-929.

28 Khoury J, Henriksen T, Christophersen B, Tonstad S. Effect of a cholesterol-lowering diet on maternal, cord, and neonatal lipids, and pregnancy outcome: a randomized clinical trial. American Journal of Obstetrics and Gynecology 2005; 193:1292-1301.

29 Lockwood CJ, Kuczynski E. Markers of risk for preterm delivery. Journal of Perinatal Medicine 1999; 27:5-20. 
30 Olsen SF, Secher NJ, Tabor A, Weber T, Walker JJ, Gluud C. Randomised clinical trials of fish oil supplementation in high risk pregnancies. Fish Oil Trials In Pregnancy (FOTIP) Team. BJOG: An International Journal of Obstetrics and Gynaecology 2000; 107:382-395.

31 Ness RB, Bodnar L, Holzman C, Platt RW, Savitz DA, Shaw GM, et al. Thoughts on the future of reproductive and perinatal epidemiology. Paediatric and Perinatal Epidemiology 2013; 27:11-19.

32 Galtier-Dereure F, Bringer J. [Obesity and pregnancy]. Annales d'Endocrinologie 2002; 63:470-475.

33 Hedderson MM, Ferrara A, Sacks DA. Gestational diabetes mellitus and lesser degrees of pregnancy hyperglycemia: association with increased risk of spontaneous preterm birth. Obstetrics and Gynecology 2003; 102:850-856.

34 Yang X, Hsu-Hage B, Zhang H, Zhang C, Zhang Y. Women with impaired glucose tolerance during pregnancy have significantly poor pregnancy outcomes. Diabetes Care 2002; 25:1619-1624.
35 Cuco G, Fernandez-Ballart J, Sala J, Viladrich C, Iranzo R, Vila J, et al. Dietary patterns and associated lifestyles in preconception, pregnancy and postpartum. European Journal of Clinical Nutrition 2006; 60:364-371.

\section{Supporting information}

Additional Supporting Information may be found in the online version of this article at the publisher's web-site:

File S1. Construction and validation of the food frequency questionnaire.

File S2. Sensitivity analyses and complementary stratified analyses taking into account weight gain during pregnancy. 